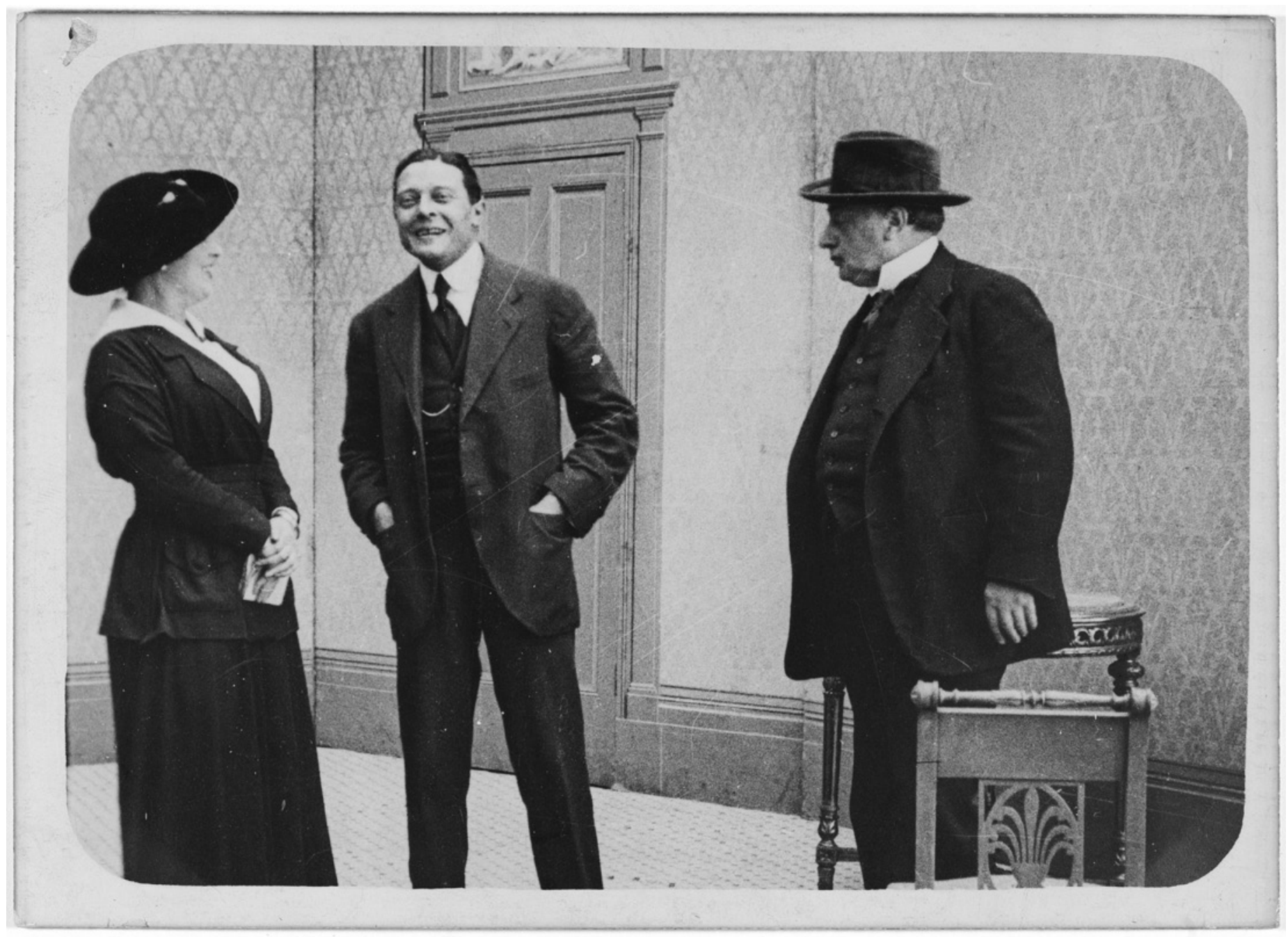

1. Ceux de chez nous, 1915

Photographie de tournage

Musée Carnavalet, CARPH041649_1

Ceux de chez nous (1915), de Sacha Guitry, constitue une véritable archive, étant souvent la seule trace mouvante de ces personnalités filmées dans leur activité. Antoine dirige ici Jane Faber et Henri Desfontaines lors d'une répétition d'une scène de L'Avare spécialement conçue pour le film. 


\title{
Un échange épistolaire pendant la guerre : André Antoine et Henri Desfontaines, de la scène à l'écran
}

MANON BILLAUT

\author{
En mai r9r4, André Antoine démissionne de l'Odéon et se lance \\ dans le cinéma. Quelques mois plus tard, la France entre \\ en guerre, et le jeune Henri Desfontaines est mobilisé au Service \\ cinématographique de l'armée. C'est ainsi le parcours de ces \\ deux hommes, qui se sont rencontrés au théâtre et retrouvés \\ dans le septième art, que nous retraçons, à travers leurs \\ échanges épistolaires, conservés dans le fonds André Antoine \\ du département des Arts du spectacle de la BNF.
}

$\mathrm{L}$ E FONDS André Antoine, conservé au département des Arts du spectacle de la Bibliothèque nationale de France, contient une volumineuse correspondance, d'environ 20000 lettres, qui constitue une véritable mine d'informations pour l'histoire du théâtre et du cinéma des années I9IOI $920^{1}$. Elle permet de comprendre le rôle éminent tenu par André Antoine, metteur en scène de théâtre, cinéaste, puis critique dramatique et cinématographique. Parmi ses plus fidèles correspondants figure Henri Desfontaines (I876-I931), né Paul-Henri Lapierre, élève et ami d'Antoine. Les quelque I20 lettres, cartes, télégrammes, pneumatiques qui ont été conservés invitent à creuser la relation entre ces deux hommes que la Première Guerre mondiale a rapprochés, I9I 4 lançant leurs carrières cinématographiques respectives ${ }^{2}$. On sait en effet que la Grande Guerre, bouleversant les conditions de la production cinématographique, constitue un moment charnière dans l'histoire du cinéma français, qui se caractérise par un renouvellement de la mise en scène, le développe- ment des techniques de prise de vues documentaires et l'élaboration de nouvelles réflexions sur le cinéma ${ }^{3}$.

L'étude et la mise en regard de la production cinématographique de Desfontaines et d'Antoine montrent comment tous deux, qui étaient intimement liés au théâtre, se retrouvèrent au cinéma unis par des conceptions artistiques communes, alimentées par la croyance en la capacité du medium à enregistrer la réalité et à en laisser une trace afin d'éduquer les générations futures.

Henri Desfontaines, disciple d'Antoine au théâtre

C'est sans doute au Conservatoire national d'art dramatique qu'Henri Desfontaines, qui y était élève, fut remarqué par André Antoine, toujours à la recherche de nouveaux talents, puisqu'il débuta sa carrière au Théâtre-Libre dans L'Assomption d'Hannelé Mattern (Hanneles Himmelfahrt), de Gerhard Hauptmann (I893), créée par André Antoine le $\mathrm{I}^{\mathrm{er}}$ février I 894. Le jeune Desfontaines y interprète le tailleur du village, qui se matérialise devant le person- 


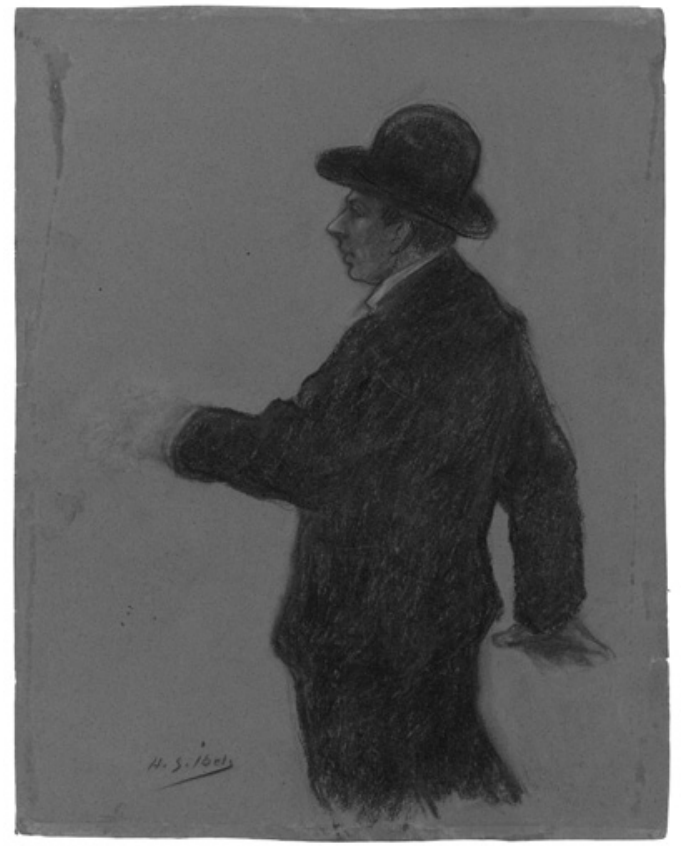

2. Henri-Gabriel Ibels, André Antoine

Dessin, pastel, $28,9 \times 22,8 \mathrm{~cm}$

BNF, Arts du spectacle, 4-0 ICO-33

Ce très beau dessin représente Antoine en mouvement, probablement en train de donner des indications scéniques. Henri Ibels a beaucoup travaillé avec Antoine, étant notamment l'un des illustrateurs fétiches des programmes du Théâtre-Libre et le décorateur de nombreuses mises en scène du maître.

nage principal dans la seconde partie de la pièce de Hauptmann. Il s'agit d'un tout petit rôle qui ne lui valut pas d'être crédité dans la distribution indiquée sur le programme ${ }^{4}$. Ce rôle, qui scelle sa première collaboration avec Antoine, a néanmoins profondément marqué Desfontaines, celui-ci y faisant à diverses reprises allusion tout au long de la correspondance, allant jusqu'à s'identifier au personnage, comme pour souhaiter ses meilleurs vœux à Antoine en I928: "Cher monsieur Antoine, pour la $34^{\mathrm{e}}$ fois, le petit tailleur sort de sa trappe pour vous apporter ses vœux ${ }^{5}$."

Deux ans après cette première expérience au Théâtre-Libre, Desfontaines décida de quitter le Conservatoire sans avoir passé le diplôme final. Venant d'apprendre qu'Antoine avait été nommé à la tête de l'Odéon, il le sollicita dans l'espoir qu'il lui ouvrirait les portes de son théâtre, comme en témoigne une petite lettre cartonnée datée du I $^{\text {er }}$ juillet I 896 demandant un entretien :

Cher monsieur Antoine, je vous prie à l'avance de m'excuser si je prends la liberté de vous écrire, mais je crois que cela est préférable, car je ne risquerai pas de vous importuner. Je viens vous demander, monsieur, de vouloir bien m'accorder un instant d'entretien en me fixant l'heure à laquelle cela ne vous dérangera pas. Je serais en effet très désireux d'entrer à l'Odéon : je quitte le Conservatoire n'ayant pas réussi à mon service d'examen. [...] En espérant que vous n'aurez pas gardé un trop mauvais souvenir du Petit tailleur d'Hannelé, veuillez agréer, monsieur, l'assurance de ma respectueuse considération ${ }^{6}$.

C'est l'échange le plus ancien entre les deux hommes conservé dans la correspondance ${ }^{7}$. Antoine venait en effet d'être nommé à la tête du second Théâtre-Français, mais il n'y resta que quelques jours, laissant la place à Paul Ginisty, le célèbre chroniqueur du journal Gil Blas, avec qui il codirigeait le théâtre $^{8}$. Il ne put donc donner suite à la demande de Desfontaines. Mais quelques mois plus tard, alors qu'il installait le Théâtre Antoine dans la salle des Menus-Plaisirs, il pensa à lui pour sa troupe. L'acteur fait ainsi partie des fidèles dès le début de l'aventure, comme le narre Antoine dans ses Mémoires :

$\mathrm{I}^{\text {er }}$ juillet I 897. J'ai signé le bail avec Mme Cantin et me voici enfin chez moi. J'ai immédiatement battu le rappel de mes anciens camarades du Théâtre-Libre et tous arrivent avec empressement. Gémier des premiers, Arquillère, Janvier, Luce Colas, Henriot, Desfontaines et d'autres. Mon ancienne troupe est reconstituée; il n'y a plus qu'à se mettre à l'ouvrage ${ }^{9}$.

De son côté, Desfontaines se dit très heureux, dès le mois d'août, de rejoindre la troupe du Théâtre Antoine :

Cher monsieur Antoine, je vous remercie sincèrement d'avoir pensé à moi pour faire partie de la troupe de votre nouveau théâtre. J'accepte avec d'autant plus de plaisir que j'étais très désireux de pouvoir encore travailler avec vous. Espérons que je pourrai vous satisfaire et le plus longtemps possible : c'est ce que je souhaite le plus. Merci encore et veuillez agréer l'assurance de ma considération ${ }^{10}$.

4 Programme conservé dans le fonds Radio France de la bibliothèque Gaston Baty sous la cote RF 3323. 5 Correspondance Antoine, Henri Desfontaines, fonds André Antoine, BNF, Arts du spectacle, 4-COL-113 (2108), 21 décembre 1928. 6 Correspondance Antoine, Henri Desfontaines, fonds André Antoine, BNF, Arts du spectacle, 4-COL-113 (2108), 1 er juillet 1896. 7 La correspondance s'étend de 1896 à 1928. 8 Antoine dirigea une première fois l'Odéon en codirection avec Paul Ginisty du 10 au 27 octobre 1896 , puis il fut nommé à nouveau à la tête du théâtre en juin 1906. II donnera sa démission en 1914. 9 André Antoine, Mes souvenirs sur le Théâtre Antoine et sur l'Odéon (première direction), Paris, France, Bernard Grasset, 1928, p. 118. 10 Correspondance Antoine, Henri Desfontaines, fonds André Antoine, BNF, Arts du spectacle, 4-COL-113 (2108), 6 août 1897. 
Cet engagement marque le début d'une relation très intime, presque paternelle, et de la longue collaboration entre les deux hommes, Desfontaines suivant Antoine à l'Odéon à partir de $1906^{11}$. C'est ainsi que, lorsqu'il se maria avec l'actrice Jeanne Grumbach, qu'il avait très probablement rencontrée sur la scène du Théâtre-Libre, le 30 octobre I9I2, Desfontaines invita Antoine au déjeuner familial, parmi le cercle restreint des proches :

J'espère bien que vous nous ferez le grand plaisir d'être des nôtres au petit déjeuner qui aura lieu ensuite chez Foyot, sans aucune cérémonie. Nous serons sept en tout : la maman, les témoins, et les mariés. Merci du fond du cœur, cher monsieur Antoine, et croyez à tout mon dévouement et à ma mineure reconnaissance ${ }^{12}$.

Dans un autre courrier non daté, mais que nous pouvons situer vers I920, Desfontaines, qui sort d'une période difficile, se réjouit de retrouver du travail en jouant pour Sacha Guitry. Il termine sa lettre par ces mots : «À bientôt, mon bien cher patron, mon "bien cher père", je sais que vous me permettez de vous donner ce nom.» Le père de substitution est ainsi nommé.

C'est à la fois son énergie, son ambition et son talent qui font apparaître Desfontaines dans la quasitotalité des pièces montées au Théâtre Antoine et à l'Odéon. Un tableau de bord de l'Odéon publié dans Comcedia en 1907 montre qu'en cent jours, du 24 septembre au 3 I décembre, Desfontaines joua huit rôles, pour un total de $8 \mathrm{I}$ représentations, ce qui est le maximum de rôles différents interprétés par un acteur pendant cette saison. Il apparaît en quatrième position derrière Léon Bernard, Erbaf (Saturnin Fabre) et Denis d'Inès. Rarement tête d'affiche, il interprète quelquefois plusieurs rôles dans une même pièce. Mais ce travail intense poussa Desfontaines à se plaindre à Antoine :

Je suis très fatigué, moralement et physiquement. Depuis le commencement de la saison, $j$ 'ai joué douze rôles, et seul de tous les camarades j'ai été de tous les spectacles du soir. Vous savez trop combien j'ai à cœur de donner l'exemple pour ne pas comprendre ma demande. Il n’y a jamais de corvée pour moi, nous devons tous travailler pour la maison, mais ce prochain rôle va encore me tenir au théâtre toutes les soirées. Si je n'étais que du premier acte je pourrais me retaper ${ }^{13}$.

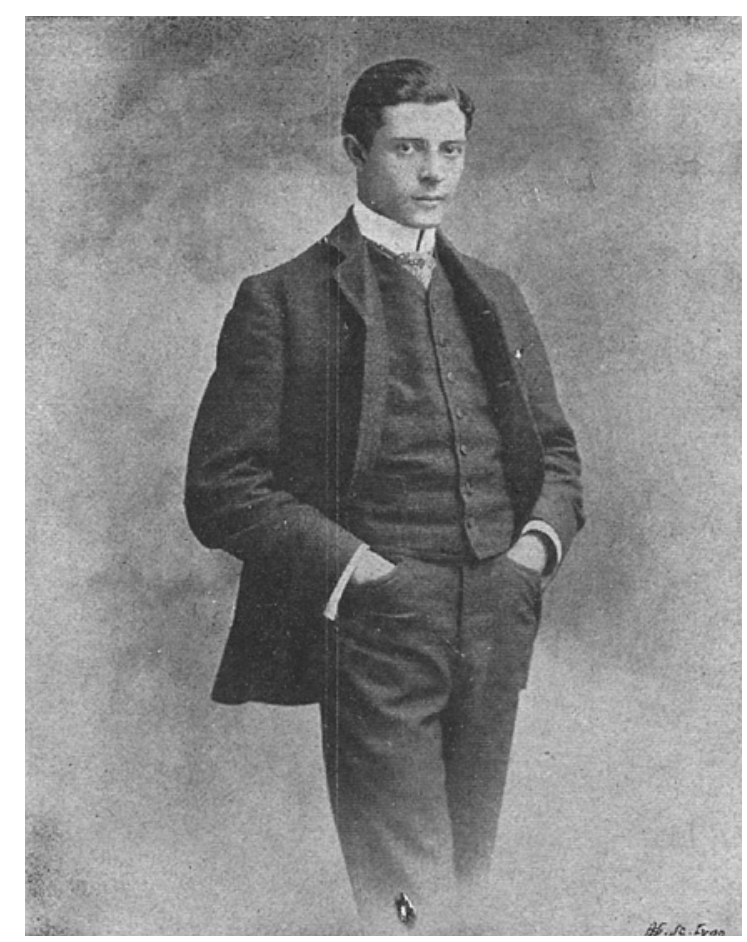

3. Portrait d'Henri Desfontaines, 1912 BNF, Arts du spectacle, 4-ICO-THE-2573 II existe peu de portraits d'Henri Desfontaines. Cette photographie figure dans le programme de l'Odéon édité pour la représentation de Faust en 1912. Desfontaines, en jeune premier, détonne parmi ses camarades, les plus connus étant même photographiés par le célèbre Nadar.

On retrouve à plusieurs reprises ce caractère plaintif dans les lettres de Desfontaines. La lettre, non datée, ne nous permet pas d'identifier de quelle pièce il s'agit, mais la requête de Desfontaines montre bien que l'acteur arrivait au bout de ses forces. Sa notoriété allait pourtant croissant. À l'Odéon, en octobre I9I I, il fut à la tête de La Mort de Sénèque, interprétant Procule, aux côtés de Romuald Joubé et de Jacques Grétillat; en avril I9I2, il eut le troisième rôle dans L'Honneur japonais, de Paul Anthelme, pour jouer le rôle d'Ouakami; et en décembre I9I2, on le vit à l'affiche de Faust, dans le rôle de Méphisto, aux côtés de Romuald Joubé, qui tenait le rôle de Faust. Cette pièce, jouée pour la première fois le 2I décembre I9I2, fut représentée tous les soirs et rapporta entre 3000 et 4000 francs de recettes par soir, ce qui est la preuve d'un grand succès ${ }^{14}$.

11 Après l'Odéon, Antoine fera de nouveau appel à Desfontaines pour lui confier le poste de régisseur du Théâtre Pigalle quand il en sera le directeur, en 1927. 12 Correspondance Antoine, Henri Desfontaines, fonds André Antoine, BNF, Arts du spectacle, 4-COL113 (2108), non datée (octobre 1912). Voir le contrat de mariage conservé au service d'état civil de la mairie du 6e arrondissement. Les deux acteurs divorceront le 18 avril 1921. 13 Correspondance Antoine, Henri Desfontaines, fonds André Antoine, BNF, Arts du spectacle, 4-COL-113 (2108), non datée. 14 Indication donnée dans le registre de l'Odéon (10 mars 1909-16 février 1913), Archives nationales, $\mathrm{AJ} / 55^{*} / 22$. 


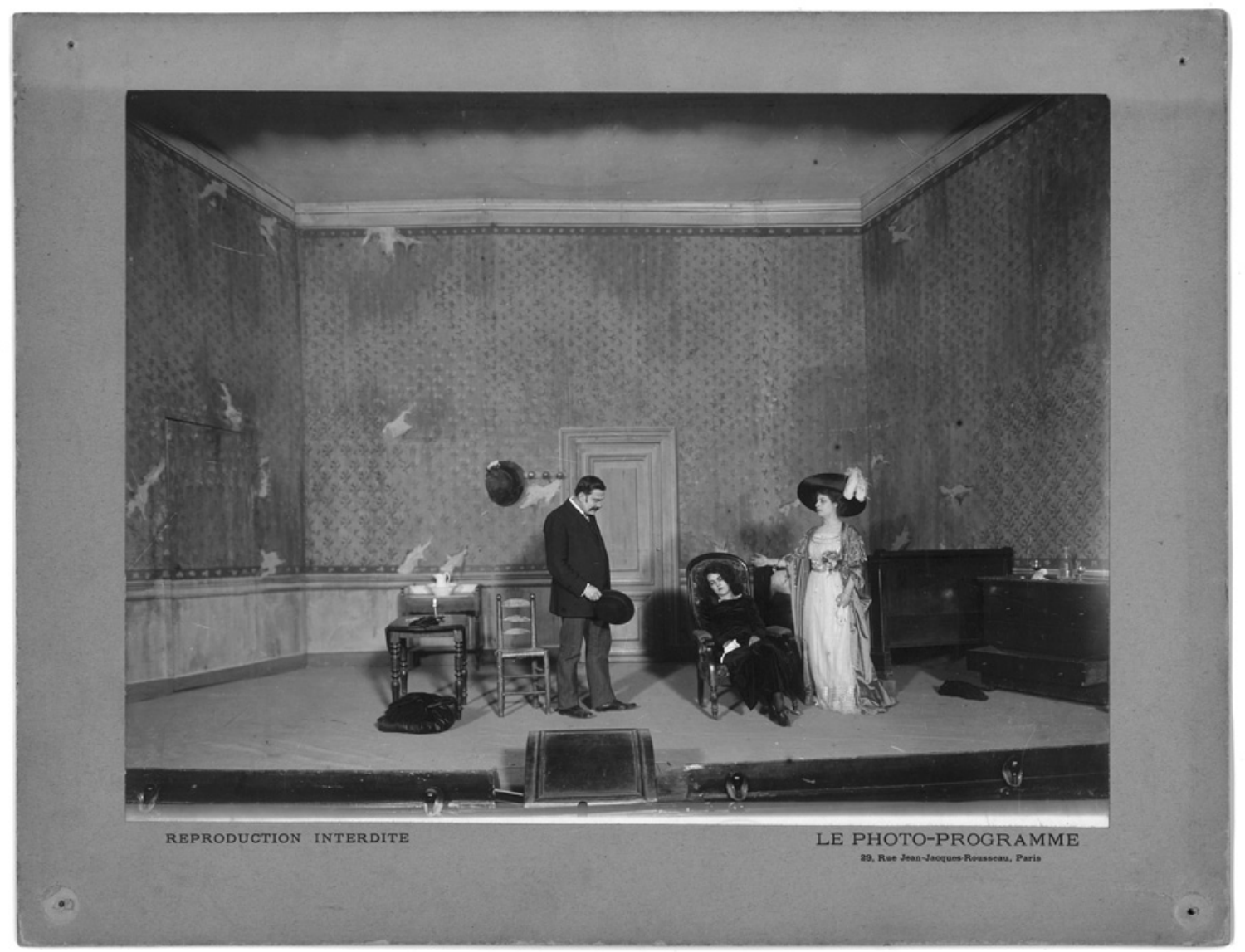

4. Prostituée, drame d'Henri Desfontaines, 1910 BNF, Arts du spectacle, FOL-ICO THE-65

Desfontaines cumulait son métier d'acteur avec celui de metteur en scène de théâtre.

Sa première mise en scène, Prostituée, eut un très grand succès. Pièce en cinq actes tirée du roman de Victor Margueritte, elle fut jouée pour la première fois le 6 avril 1910 au Théâtre de l'Ambigu.

Le physique élancé de Desfontaines lui permettait d'interpréter divers types de rôle, et il n'hésitait pas à se vieillir pour se fondre dans des personnages plus âgés, notamment dans le rôle du vieil Ekdal dans Le Canard sauvage, pièce d'Ibsen créée à l'Odéon en I9I3; ou encore Picurent dans La Rue du Sentier, de Pierre Decourcelle et André Maurel, la même année. Antoine releva d'ailleurs cette aptitude au travestissement dans l'article qu'il lui consacrera à sa mort, survenue en I93I :

Henri Desfontaines, qui vient de disparaître subitement, l'un de mes plus anciens compagnons de labeur, [...] depuis I 888, où il débuta au Théâtre-Libre, a joué d'innombrables rôles classiques et modernes, au Théâtre Antoine et à l'Odéon, où il se signala par de curieuses interprétations. Il était, comme Signoret et Gémier, l'un de nos comédiens les plus habiles à se transformer ${ }^{15}$.
Le chroniqueur avait pour habitude d'écrire une petite notice nécrologique dans sa rubrique du fournal quand la mort touchait l'un de ceux qu'il avait particulièrement côtoyés. Ces quelques lignes sont néanmoins plus personnelles que d'ordinaire, Antoine parlant de sa propre expérience de metteur en scène et désignant Desfontaines comme «l'un de [s]es plus anciens compagnons de labeur".

Compagnon d'Antoine, Desfontaines ne fut pas seulement son disciple, il partagea avec lui d'authentiques questions de mise en scène, au théâtre comme au cinéma.

\section{Un élan artistique partagé vers le cinéma}

Comme Antoine, Desfontaines alliait en effet une carrière dramatique à une carrière cinématographique. Mais si Antoine abandonna le théâtre pour se 
consacrer au cinéma en I9I4, Desfontaines cumula les rôles, étant à la fois acteur de théâtre, de cinéma aussi bien que metteur en scène sur les planches et à l'écran. Sa carrière cinématographique fut beaucoup plus importante que celle d'Antoine : Desfontaines tourna son premier film, Hamlet, en I 908 pour la société de production Éclipse, et son dernier, Le Film $d u$ poilu, produit par P. J. de Venloo, en I927, avec en tout environ une soixantaine de films. Antoine eut une carrière plus condensée, tournant neuf films entre I9I 5 et I922. Desfontaines continua également à jouer au cinéma comme au théâtre, tournant dans le film d'Henri Fescourt La Maison de la flèche (I930) peu de temps avant sa mort.

Dès les années I900, il demandait régulièrement à Antoine l'autorisation de s'échapper de l'Odéon afin d'aller tourner quelques scènes au "théâtre» de prises de vues, c'est-à-dire aux studios de la Société cinématographique des auteurs et gens de lettres (SCAGL) à Vincennes, prétextant le plus souvent une nécessité financière :

Cher monsieur Antoine, le cinéma des Gens de lettres me demande pour faire une bande la semaine prochaine. Mais exceptionnellement ils auraient besoin de moi un après-midi. Si cela ne dérange pas le service, pourriez-vous me donner votre autorisation et je leur rendrai la réponse qu'ils attendent. Il y a quelques louis à gagner. Veuillez je vous prie me croire votre très respectueux et reconnaissant Henri Desfontaines ${ }^{16}$.

C'est ainsi d'abord en tant qu'acteur qu'il fut engagé à la SCAGL, pour jouer notamment dans les films d'Albert Capellani, directeur artistique de la firme et l'un des cinéastes les plus importants du muet français ${ }^{17}$. Il y retrouva des acteurs du ThéâtreLibre d'Antoine, comme sa future femme, Jeanne Grumbach, dans L'Arlésienne ( I908), d'Albert Capellani, Jacques Grétillat dans L'Homme aux gants blancs (I908), d'Albert Capellani, et le frère de ce dernier, Paul, dans La Peau de chagrin (I909), de Michel Carré.

Il gravit rapidement les échelons et passa derrière la caméra peu avant la guerre. En I9I3, il s'associa à Paul Garbagni pour tourner La Carabine de la mort, s'entourant lui aussi des fidèles du Théâtre-Libre : Romuald Joubé, Jacques Grétillat, Jeanne Grumbach, Maxime Desjardins. La même année, il tourna seul
Le Secret de Polichinelle d'après une pièce de Pierre Wolff représentée pour la première fois au Théâtre du Gymnase le 5 janvier I903. Face au succès grandissant du cinéma et aux déceptions accumulées au théâtre, il songea à abandonner sa carrière dramatique pour se consacrer au cinéma, comme le prouve une lettre non datée mais que nous pouvons situer vers I9I3, qu'il adresse à Antoine après avoir été refusé à la Comédie-Française :

J'ai donc raté le Théâtre-Français! Je n’insiste plus! J'attendrai la fin de la saison et puis adieu le théâtre où je n'ai rencontré que tristesses après avoir travaillé comme peu d'artistes. Je vais refaire ma vie ${ }^{18}$ !

L'entrée en guerre de la France précipita les choses. En avril I9I4, Antoine démissionna de l'Odéon, n'ayant pu récolter les subventions nécessaires pour combler l'extrême faillite du théâtre. Par nécessité, Desfontaines ne put pas démissionner, même s'il eût probablement voulu manifester sa solidarité envers son maître et ami en quittant le théâtre avec lui. Un mois plus tard, la saison reprit à l'Odéon sous la direction de Paul Gavault, et Desfontaines se retrouva à l'affiche des Saltimbanques, comédieparade de Théophile Dumersan et Varin (Charles Voirin), le 30 mai I9I $4{ }^{19}$. Mais dès août I9I4 la

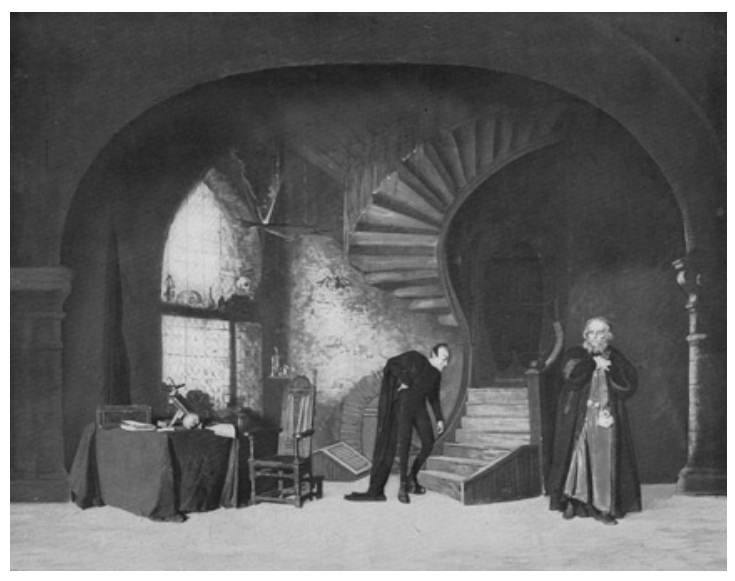

5. Desfontaines dans Faust, 1912 BNF, Arts du spectacle, 4-ICO-THE-2573

Desfontaines dans Faust, mis en scène pour la première fois par André Antoine à l'Odéon le 21 décembre 1912. «M. Desfontaines a fait de Méphisto une création particulièrement intéressante, c'est le bon diable, ironique, spirituel, lecteur averti de Voltaire», Jean Renouard, dans Le Théâtre. 


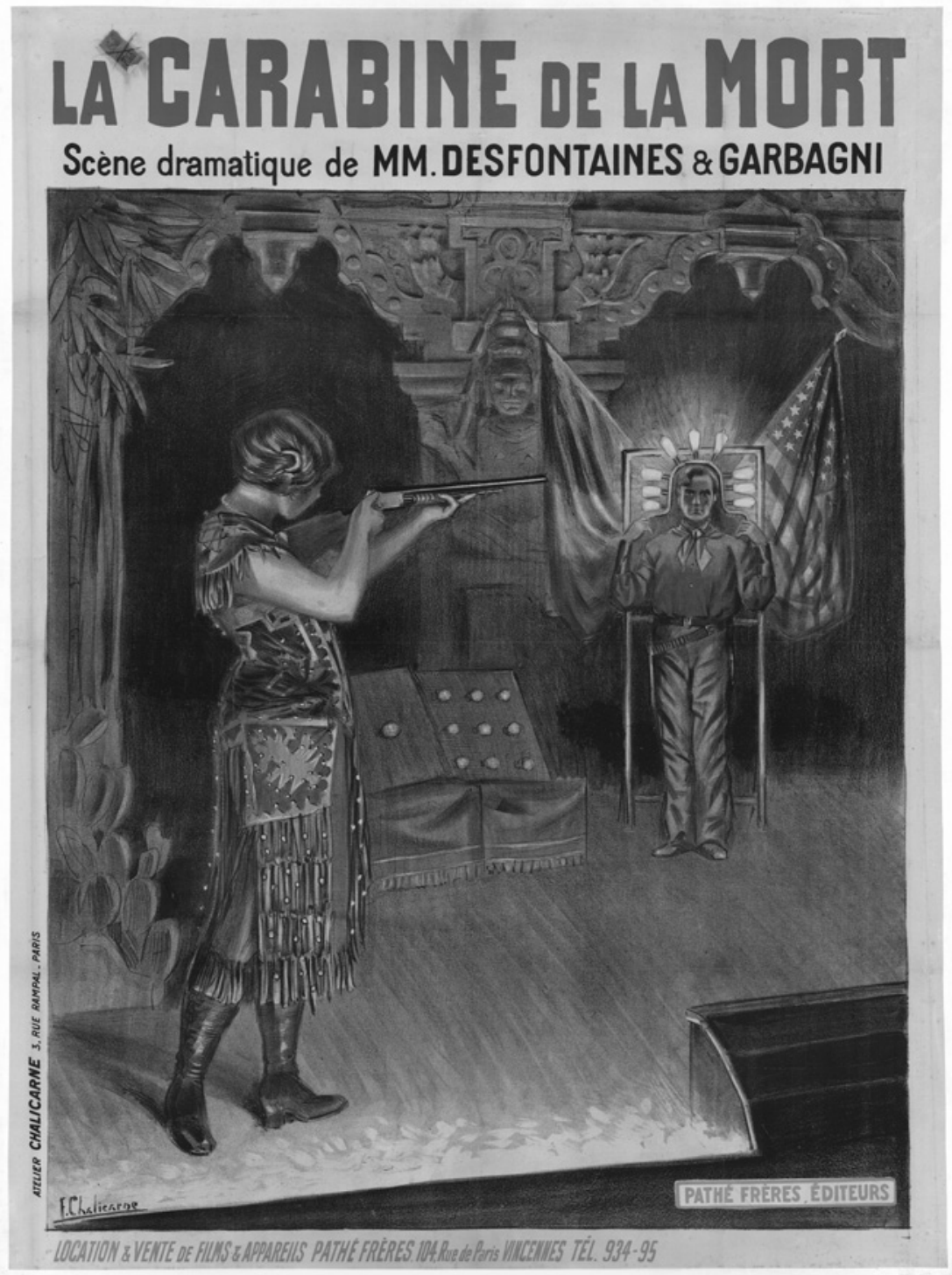

6. Chalicarne, La Carabine de la mort, 1913

Affiche, lithographie, $160 \times 120 \mathrm{~cm}$ Fondation Jérôme Seydoux-Pathé, AFF-P-00001503

L'affiche de La Carabine de la mort (1913),

d'Henri Desfontaines et de Paul Garbagni, présente l'invention créée par l'ingénieur Karl pour les Folies Palace. Musidora, armée de sa carabine, tire sur Fred Curtis

(Romuald Joubé), et grâce à un système électrique crée une auréole lumineuse autour de la cible. mobilisation générale emmena le nouveau directeur sur le front et le théâtre ferma ses portes ${ }^{20}$. De son côté, Antoine répondit favorablement aux propositions de Pierre Decourcelle et d'Eugène Gugenheim et rejoignit la SCAGL en tant que metteur en scène cinématographique. La guerre retarda l'entreprise, et il ne put commencer à tourner véritablement son premier film, Les Frères corses, qu'en I9I6, après un séjour de deux mois à Istanbul, où il avait été appelé pour fonder le Théâtre national ottoman.
À l'été I9I5, il participa au projet de son ami Sacha Guitry, qui avait entrepris de filmer les grands hommes du début du $\mathrm{xx}^{\mathrm{e}}$ siècle dans leur activité. Le documentaire, intitulé Ceux de chez nous, porté par une mission de mémoire nationale, était accompagné d'un commentaire que Guitry prononçait lui-même en direct, comme un bonimenteur, lors des nombreuses représentations ${ }^{21}$. Il demanda à Antoine de mimer une mise en scène pour ce petit film encyclopédique, et celui-ci fit appel à Jane Faber et à Desfon-

20 Voir le registre de l'Odéon (7 mai 1914-25 avril 1918), Archives nationales, AJ 55* 24. 21 Une version de vingt-deux minutes fut en effet présentée pour la première fois à Paris le 22 novembre 1915 au Théâtre des Variétés, sous le titre Ceux de chez nous. Cette première séance ne rassembla que quelques privilégiés, des officiels et certains des artistes filmés. Le spectacle fut ensuite repris de manière régulière en décembre 1915 au Théâtre du Palais-Royal. Voir entre autres Alain Carou, «Ceux de chez nous : galerie de portraits, théâtres de la mémoire », dans Noëlle Giret, Noël Herpe (dir.), Sacha Guitry : une vie d'artiste (exposition, Paris, Cinémathèque française, 17 octobre 2007-18 février 2008), Paris, Gallimard, 2007. 
taines, qui était à Paris, pour jouer la scène ${ }^{22}$. Un pneumatique de l'acteur envoyé à Antoine et daté du 11 juillet I9I 5 confirme sa venue :

Cher monsieur Antoine, vous pouvez certainement compter sur moi lundi. Je serai à 3 heures chez Pathé au théâtre rue du Bois. J'ai téléphoné à Faber qui viendra également : elle a joué le rôle, vous vous rappelez. Si c'est possible, je vous demanderai d'être libre à 4 heures $1 / 2$. Veuillez agréer, cher monsieur Antoine, l'expression de mon dévouement.

Dans le film, on voit d'abord Antoine filmé dans la propriété de Guitry à Yainville-Jumièges, puis en intérieur. Il dirige ses acteurs, en mimant une répétition d'une scène de L'Avare spécialement conçue pour le film, allant même jusqu'à reproduire luimême le jeu désiré, la fausse répétition devenant réalité sous nos yeux ${ }^{23}$. Antoine croyait fermement aux capacités du cinéma comme forme d'expression moderne, et ce projet documentaire de Guitry lui plut grandement. Dans une lettre à son fils, il loua ainsi le projet ambitieux de son ami : «Il entremêle les choses de dialogues de lui et l'ensemble est neuf et curieux. Il aura un succès fou avec ça ${ }^{24}$."

Antoine et Desfontaines partageaient l'idée que le cinéma pouvait éduquer les foules et être un des médias par lesquels s'exercerait un travail de mémoire. Ceux de chez nous est pourtant le seul projet cinématographique qui ait réuni les deux hommes.

\section{Les archives cinématographiques de l'armée}

Recruté par la Section cinématographique de l'armée (SCA), Desfontaines développa ses qualités de metteur en scène sous les drapeaux ${ }^{25}$. En effet, dès l'entrée en guerre de la France, une majorité des cinéastes en exercice mirent leur talent au service de la nation en réalisant des films patriotiques. De plus, les maisons de production voyaient dans ces images tournées au front et fournies par l'armée une forme d'attraction pour le public à l'arrière, ce qui constituait une motivation économique supplémentaire.

Au cours du premier semestre I9I5, deux struc- tures chargées de prendre des vues de guerre furent créées, la Section photographique de l'armée (SPA) et la Section cinématographique de l'armée (SCA), afin de satisfaire les exigences d'information dans le pays et de propagande à l'étranger. La SCA regroupait plusieurs opérateurs mobilisés appartenant dans le civil aux grandes firmes cinématographiques. Leur mission était double : mener une propagande à partir d'images authentiques et rassembler des archives cinématographiques. En janvier 1917, dans un vrai souci de production, la SCA fusionna avec la SPA afin de donner naissance à la SPCA, placée sous la tutelle conjointe des ministères de la Guerre et des Beaux-arts. C'est à ce moment-là que les projets ambitieux de Desfontaines, développant un style hybride, mêlant le documentaire à la fiction, virent le jour. Au mois de novembre sortit sur les écrans $L a$ Puissance militaire de la France, montrant l'effort de guerre à l'avant et à l'arrière. Desfontaines avait mis à profit les images enregistrées par la SCA pendant trois ans, en les mêlant à des images de fiction, afin de montrer l'investissement de la France pendant la guerre. L'impressionnant montage était destiné à convaincre le public que la nation française maîtrisait parfaitement la situation. Contribuant à former la notion d'images d'archives chère à Antoine, ce film eut une portée considérable, en France et à l'étranger, où il fut largement diffusé ${ }^{26}$. Émile Vuillermoz, critique cinématographique dans le journal Le Temps, parla même d'une nouvelle écriture de l'histoire :

Car le cinéma, sans altérer en rien la vérité, est le plus merveilleux des conteurs, et l'on sent bien qu'il faudrait un poète et un historien de génie pour donner de la guerre un raccourci aussi puissant et aussi émouvant que ce récit sincère et bref d'un témoin oculaire qui ne fait pas de littérature ${ }^{27}$ !

Dans une lettre postérieure à la réalisation du film, Desfontaines expliqua à Antoine ce qui lui avait été demandé par les services de propagande :

Pour La Puissance militaire de la France, il nous était recommandé de montrer des diagrammes de produc-

22 Desfontaines avait été renvoyé dans ses foyers le 28 février 1915. II fut ensuite appelé le 3 mai 1915 mais ne prit ses fonctions

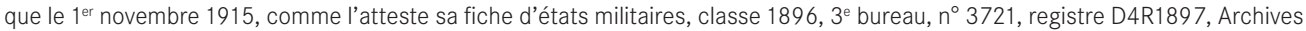
de Paris. 23 Selon les différentes versions manuscrites et dactylographiées du commentaire de 1915 (fonds Sacha Guitry, BNF, Arts du spectacle, cote : Ceux de chez nous SP 32), il s'agit bien de L'Avare et non de L'École des femmes, comme l'indique par erreur Sacha Guitry dans son commentaire enregistré dans la version de 1952, affirmation généralement reprise par les chercheurs (voir entre autres Alain Carou, «Ceux de chez nous... », dans Noëlle Giret, Noël Herpe (dir.), Sacha Guitry...,op. cit.). 24 Correspondance Antoine, André Antoine à André-Paul, Fonds André Antoine, BNF, Arts du spectacle, 12 juillet 1915. 25 Classé dans les services auxiliaires en 1899 pour faiblesse, Desfontaines fut mobilisé dès le 3 août 1914 puis libéré provisoirement le 6 décembre 1914. II fut ensuite convoqué le 25 janvier 1915 à la 20e section des secrétaires d'état-major puis passa dans la réserve de l'armée territoriale le $1^{\text {er }}$ octobre 1917 mais sera à plusieurs reprises placé en sursis d'appel, jusqu'à la fin de la guerre. Voir fiche d'états militaires, Lapierre Paul, Henri, classe 1896, 3e bureau, no 3721, registre D4R1897, Archives de Paris. Concernant son affectation à la SCA, voir Laurent Véray, Les Films d'actualité français de la Grande Guerre, AFRHC, Paris, 1995, p. 108. 26 Voir Laurent Véray, La Grande Guerre au cinéma..., op. cit., p. 16-20. 27 Émile Vuillermoz, "La puissance militaire de la France», Le Temps, 26 septembre 1917. 
tion, d'indiquer la portée des différents canons, les poids des projectiles etc. On ne doit pas, pour le but particulier à atteindre, envisager en ce moment le cinéma au point de vue général. [...] L'avenir n'est pas inquiétant pour notre art cinématographique mais, pour le présent, la propagande se fait et doit se faire avec des cartes postales et non avec des œuvres d'art... autrement le but n'est pas atteint, c'est-à-dire de pénétrer dans des classes simples.... On les éduquera peu à peu; à petites doses ${ }^{28}$.

Malgré ces contraintes, les œuvres réalisées par Desfontaines trouvèrent dans cette combinaison d'images d'archives et de fiction une nouvelle forme d'expression contribuant à l'évolution du langage cinématographique. Ayant ainsi défini son style, Desfontaines renouvela l'opération un an plus tard avec Les Enfants de France pendant la guerre, proposant un assemblage de vues tournées pendant le conflit et réorganisées les unes par rapport aux autres au moyen d'une trame narrative fictionnelle. Un soldat en permission rentrant chez lui pour la naissance de son fils offre à sa fille Micheline un album sur le rôle des enfants pendant la guerre, qui s'anime, faisant ainsi défiler les vues de la SCA. Les vues documentaires prennent ensuite le pas sur la fiction, mettant en valeur les images authentiques de la guerre. Dans la lignée de ces productions patriotiques, Desfontaines eut également le projet de monter avec Antoine un spectacle alliant des images d'archives, de fiction et une conférence :

Cher monsieur Antoine, que penseriez-vous de ce projet : une tournée de propagande : France, Espagne, Angleterre, Amérique, Égypte, Japon, etc. etc. avec ce programme : conférence par $M$. Antoine, petites comédies d'actualité ou autres, projection de films de la Section Cinéma, etc., etc... Vous, Grumbach, moi, un opérateur. Nous vivrons de nos recettes : représentations dans les salles, dans les mairies, n'importe où. Abandon d'une partie des recettes à des œuvres de guerre. Il faut juste quelques billets de mille pour les premiers jours... ça se trouve. Je suis convaincu qu'on ferait là un travail intéressant. Rien ne va plus en ce moment : théâtres, cinémas, battent de l'aile, mais en province, en revanche!! Un Baret, un Dufrenne, ferait l'itinéraire avec un pourcentage qu'on leur laisserait. J'espère que vous voudrez bien me donner votre avis, et veuillez agréer, cher monsieur Antoine, l'expression de mon respectueux dévouement ${ }^{29}$.
La lettre détaille précisément l'entreprise, centrée sur la valorisation de l'image d'archive et l'éducation du public. Malheureusement, ce projet que nous pouvons situer approximativement à l'immédiat après-guerre ne se réalisa pas, Antoine étant peut-être trop âgé pour se lancer dans une telle aventure ${ }^{30}$. Après son dernier film, L'Arlésienne (I922), Antoine se consacra désormais exclusivement à la critique cinématographique. S’il ne mit pas lui-même en pratique ce genre de films hybrides, il contribua à le théoriser dans ses articles et à faire prendre conscience de la valeur de l'image cinématographique en tant qu'archive. Il rejoint Desfontaines sur la valeur des images comme traces authentiques du passé et se révèle aussi très sensible à leur conservation et à leur valorisation $^{31}$. Selon lui, les archives cinématographiques pouvaient contribuer à l'édification d'un véritable "monument de propagande et d'éducation populaire ${ }^{32}$ ». Ainsi, il s'insurgea en I923, alors qu'on s'apprêtait à porter à l'écran L'Histoire de France, contre les mauvaises conditions de conservation des images tournées par la SCA pendant la guerre :

On sait que le Service photographique de l'armée a durant les hostilités fait tourner sur le front même, et souvent au milieu des combattants, des milliers et des milliers de vues qui, mises en ordre et disposées congrûment, constitueraient pour l'avenir la plus authentique chronique de l'Épopée. Pour des raisons faciles à comprendre, toutes ces images, des tonnes de pellicules, ne furent jamais projetées en public pendant la guerre. On les a conservées dans des archives spéciales, mais le temps qui mange la pellicule va les anéantir. Il dort là, pourtant, toute la tragédie magnifique et monstrueuse, les combattants, les paysages, la nature convulsée, la souffrance des hommes, la vérité et la vie ardente d'une époque unique. Ne serait-il pas temps d'en assurer la conservation et d'en tirer, pendant qu'il en est temps encore, des pages capitales de notre Histoire contemporaine ${ }^{33}$ ?

Quelques jours plus tard, il corrigea son article, ayant appris que les archives étaient en fait conservées par la Société anonyme des archives photographiques d'art et d'histoire, qui s'en était vu concéder l'exploitation par le ministère des Beaux-arts, et qu'elles étaient consultables sur rendez-vous. Cette information ne le rassura pas pour autant, lui qui

28 Correspondance Antoine, Henri Desfontaines, fonds André Antoine, BNF, Arts du spectacle, 4-COL-113 (2108), 6 juillet 1918. 29 Correspondance Antoine, Henri Desfontaines, fonds André Antoine, BNF, Arts du spectacle, 4-COL-113 (2108), non datée. 30 Desfontaines réside alors rue Pergolèse un logement qu'il occupera jusqu'en février 1919, quand il déménagera rue de Valois. 31 Concernant la reconnaissance du rôle du cinéma en tant que témoin privilégié de l'époque et source historique et d'éducation morale pour les générations futures, voir Laurent Véray, Les Images d'archives face à l'histoire : de la conservation à la création, France, SCÉRÉN-CNDP-CRDP, 2011, p. 45-52. 32 André Antoine, "Trésors enfouis», Le Journal, 26 juillet 1923.33 Ibid. 


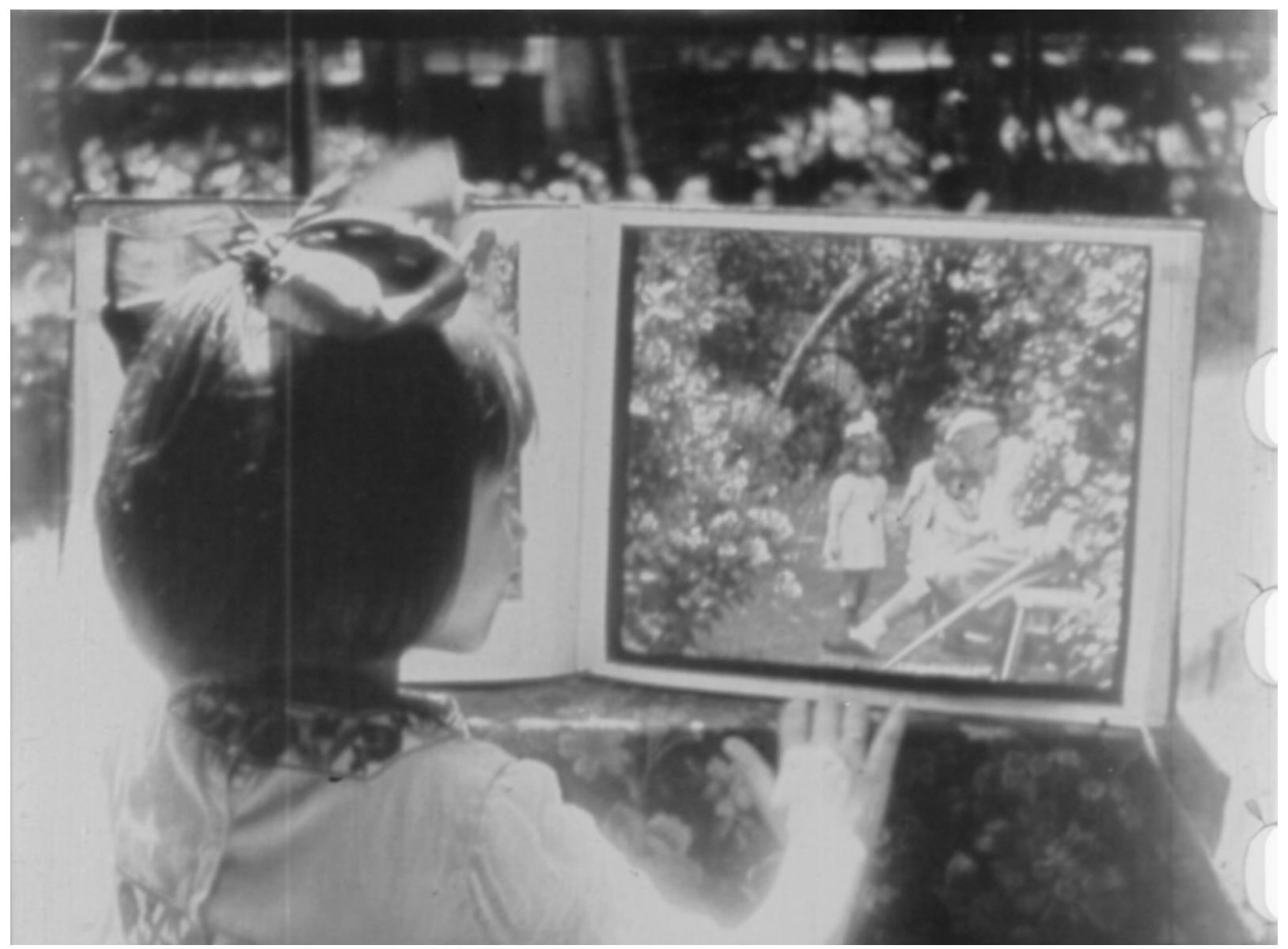

7. Photogramme extrait d'un des films de Desfontaines réalisé pendant la période SCA Voir ECPAD

aurait voulu que ce service fût public, comme l'ensemble des archives de l'État ${ }^{34}$. Sa conception très moderne de l'usage des archives filmées le poussa à envisager de remonter les vues prises auprès des armées combattantes en période de commémoration, et notamment dans la perspective du centenaire de la Première Guerre mondiale. D'où cette proposition, dans sa rubrique de Comcedia, publiée en janvier I923, concernant l'édification d'une bibliothèque cinématographique :

Songez à l'émotion du public du centenaire de 20I4, en revoyant certains âges de la récente épopée, par exemple cette revue du front, passée par le général Gouraud, où les drapeaux de l'armée victorieuse frissonnaient inoubliablement dans un pays encore tout chaud de la bataille ${ }^{35}$.
C'est donc vers le cinéma qu'Antoine, conscient de la charge émotionnelle de ces images, se tourna pour préserver et transmettre la mémoire de la Grande Guerre, tout comme Henri Desfontaines, qui, par ses «illustrations cinématographiques ${ }^{36}$ ", avait déjà contribué à porter à l'écran une vision rétrospective du terrible conflit. Unis par une même passion du théâtre, Antoine et Desfontaines se sont retrouvés au cinéma, développant une conception commune de l'image animée comme archive majeure du monde contemporain. L'inscription des images du passé dans le présent en exploitant les possibilités techniques offertes par le nouveau médium cinématographique constituait un projet mémoriel ambitieux. 